\title{
Weak interaction processes in astrophysics: neutrino winds from proto-neutron stars
}

\author{
Gabriel Martínez-Pinedo ${ }^{* 1,2}$, T. Fischer ${ }^{2,1}$, A. Lohs ${ }^{1}$, L. Huther ${ }^{1}$ \\ ${ }^{1}$ Institut für Kernphysik, Technische Universität Darmstadt, Schlossgartenstraße 2, 64289 \\ Darmstadt, Germany \\ ${ }^{2}$ GSI Helmholtzzentrum für Schwerioneneforschung, Planckstraße 1, 64291 Darmstadt, \\ Germany
}

\begin{abstract}
We show that a treatment of charged-current neutrino interactions in hot and dense matter that is consistent with the nuclear equation of state has a strong impact on the spectra of the neutrinos emitted during the deleptonization period of a protoneutron star formed in a core-collapse supernova. We compare results of simulations including and neglecting mean field effects on the neutrino opacities. Their inclusion reduces the luminosities of all neutrino flavors and enhances the spectral differences between electron neutrino and antineutrino. The magnitude of the difference depends on the equation of state and in particular on the symmetry energy at sub-nuclear densities. These modifications reduce the proton-to-nucleon ratio of the neutrino-driven outflow, increasing slightly their entropy. They are expected to have a substantial impact on the nucleosynthesis in neutrino-driven winds, even though they do not result in conditions that favor an r-process. Contrary to previous findings, our simulations show that the spectra of electron neutrinos remain substantially different from those of other (anti)neutrino flavors during the entire deleptonization phase of the protoneutron star. The obtained luminosity and spectral changes are also expected to have important consequences for neutrino flavor oscillations and neutrino detection on Earth.
\end{abstract}

XII International Symposium on Nuclei in the Cosmos,

August 5-12, 2012

Cairns, Australia

${ }^{*}$ Speaker. 


\section{Introduction}

Supernova explosions of massive stars are related to the birth of neutron stars due to the collapse of the stellar core at the end of stellar evolution [1]. The detection of neutrinos from SN1987A [2, 3] confirmed that the $\approx 3 \times 10^{53}$ ergs of gravitational energy gained by the core collapse are emitted as neutrino radiation on time scales of tens of seconds, during which the central protoneutron star (PNS) cools, deleptonizes and contracts [4]. In the delayed neutrino-heating explosion mechanism [1,5], neutrinos also transport energy from the nascent PNS to the stalled bounce shock. This mechanism remains the most viable scenario to explain supernova explosions as confirmed by recent two-dimensional simulations [6]. Once the explosion sets in, the continuous emission of neutrinos from the PNS drives a low-mass outflow known as neutrino-driven wind $[7,8]$ that is currently considered the favored site for the productions of elements heavier than iron (e.g. [9]). As neutrinos travel through the stellar mantle, they can suffer flavor oscillations [10], contribute to the nucleosynthesis of several rare isotopes [11,12] and even drive an $r$ process in the He-shell of metal-poor stars [13] before they are finally detected on Earth.

Accounting for all aspects discussed above requires the knowledge of the spectra of the neutrinos emitted during the cooling phase of the PNS. Due to their low energies $v_{\mu, \tau}, \bar{v}_{\mu, \tau}$ interact only via neutral-current reactions. Hence, together with the neutron-richness of the PNS surface one expects the following neutrino-energy hierarchy: $\left.\left.\left\langle E_{\nu_{\mu, \tau}}\right\rangle\right\rangle\left\langle E_{\bar{\nu}_{e}}\right\rangle\right\rangle\left\langle E_{v_{e}}\right\rangle[14,15]$, with $\langle E\rangle$ the average energy of the neutrino spectrum. Early supernova models $[16,17]$ predicted large energy differences between $\bar{v}_{e}$ and $v_{e}$ that resulted in neutron-rich ejecta as required by $r$-process nucleosynthesis [18]. However, as the treatment of neutrino transport and weak interaction processes improved, the computed energy difference between $\bar{v}_{e}$ and $v_{e}$ decreased and the early wind ejecta became proton rich $[19,20]$. More recently, it has been possible to perform supernova simulations based on three-flavor Boltzmann neutrino transport for time scales of several tens of seconds [21, 22], covering the whole deleptonization of the PNS. These simulations predict a continuous decrease of the energy difference between neutrinos and antineutrinos of all flavors that became practically indistinguishable after $\approx 10 \mathrm{~s}$. The exact value depends on the progenitor. The proton-richness of the ejecta increases continuously with time and leaves the $v p$ process $[23,24,25]$ as the only mechanism for producing elements heavier than iron in neutrino-driven winds.

\section{Charged-current neutrino processes at high densities}

With the development of three-flavor Boltzmann neutrino transport codes it has been possible to relate the spectra of the emitted neutrinos and the underlying nucleosynthesis to high density neutrino matter interactions and basic properties of the nuclear equation of state. The simulations of ref. [22] have been recently analyzed, showing that the convergence of neutrino and antineutrino spectra at late times is due to the suppression of charged-current processes at high densities [15]. This analysis was based on a set of neutrino opacities that assume a non-interacting gas of nucleons and nuclei. This approximation may be valid during the accretion phase prior to the onset of the supernova explosion when the region from where neutrinos decouple, the neutrinospheres, is located at relatively low densities, $\sim 10^{11} \mathrm{~g} \mathrm{~cm}^{-3}$. However, as the PNS cools the neutrinospheres move to increasingly higher densities where the non-interacting gas approximation breaks down. 
The nuclear interaction is treated at the mean-field level in equations of state (EoS) commonly used in core-collapse supernova simulations [26, 27]. However, its influence on weak interaction processes is often neglected. These EoS treat protons and neutrons as a gas of quasi-particles that move in a mean-field single particle potential $U$. Assuming non-relativistic nucleons, which is a good approximation for densities $\rho \leq 5 \rho_{0}$ where $\rho_{0} \approx 2.5 \times 10^{14} \mathrm{~g} \mathrm{~cm}^{-3}$, the energy momentum relation closely resembles the non-interacting case [28]:

$$
E_{i}\left(\boldsymbol{p}_{i}\right)=\frac{\boldsymbol{p}_{i}^{2}}{2 m_{i}^{*}}+m_{i}+U_{i}, \quad i=n, p,
$$

with particle rest-masses $m_{i}$. Both the single-particle mean-field potentials and the (Landau) effective masses, $m_{i}^{*}$ depend on density, temperature and proton-to-nucleon ratio, $Y_{e}$. Importantly, due to the extreme neutron-rich conditions the mean-field potentials for neutron and protons can be very different with their relative difference $U_{n}-U_{p}$ directly related to the nuclear symmetry energy [28] (see below).

In order to quantify the impact of the mean field potentials, let us consider (anti)neutrino absorption on (protons) neutrons. Assuming zero momentum transfer, i.e. $\boldsymbol{p}_{n} \approx \boldsymbol{p}_{p}$ (elastic approximation), the electron(positron) and (anti)neutrino energies are related by:

$$
\begin{aligned}
& E_{v_{e}}=E_{e^{-}}-\left(m_{n}-m_{p}\right)-\left(U_{n}-U_{p}\right), \\
& E_{\bar{v}_{e}}=E_{e^{+}}+\left(m_{n}-m_{p}\right)+\left(U_{n}-U_{p}\right) .
\end{aligned}
$$

Eqs. (2.2) and (2.3) show that the contribution of the mean field potential reduces (increases) the energy of the emitted neutrinos (antineutrinos). The energy difference between neutrinos and antineutrinos is increased by an amount $2\left(U_{n}-U_{p}\right)$. The opacity, or inverse mean-free path, for (anti)neutrino absorption also changes. In the elastic approximation and assuming extreme relativistic electrons, the opacity for neutrino absorption becomes [28, 29]:

$$
\begin{aligned}
\chi\left(E_{v_{e}}\right)= & \frac{G_{F}^{2} V_{u d}^{2}}{\pi(\hbar c)^{4}}\left(g_{V}^{2}+3 g_{A}^{2}\right) \times \\
& E_{e}^{2}\left[1-f_{e}\left(E_{e}\right)\right] \frac{n_{n}-n_{p}}{1-e^{\beta\left(\eta_{p}-U_{p}-\eta_{n}+U_{n}\right)}},
\end{aligned}
$$

with $E_{v_{e}}$ and $E_{e}$ related by equation (2.2). $G_{F}$ is the Fermi coupling constant, $V_{u d}$ is the up-down entry of the Cabibbo-Kobayashi-Maskawa matrix, $g_{V}$ and $g_{A}$ are vector and axial-vector coupling constants and $n_{p, n}$ the number density of protons or neutrons. $f$ is the Fermi-Dirac distribution function and $\eta$ is the chemical potential (without rest mass). The emissivity, $j\left(E_{v_{e}}\right)$, can be obtained from the detailed balance condition $j\left(E_{v_{e}}\right)=\exp \left(-\beta\left(E_{v_{e}}-\mu_{v}^{\mathrm{eq}}\right)\right) \chi\left(E_{v_{e}}\right)$, with $\mu_{v}^{\mathrm{eq}}=\mu_{e}-\left(\mu_{n}-\mu_{p}\right)$ the equilibrium neutrino chemical potential, $\mu$ the chemical potential including rest mass and $\beta$ the inverse temperature. The opacity and emissivity for antineutrino absorption are obtained by exchanging neutron and proton and using equation (2.3) to relate the positron and antineutrino energies. The quantity $\varphi=\eta-U$ represents the chemical potential for a non-interacting gas of nucleons, that is related to the nucleon number density by the relation:

$$
n=2 \int \frac{d^{3} \boldsymbol{p}}{(2 \pi \hbar c)^{3}} \frac{1}{e^{\beta\left(\frac{p^{2}}{2 m}-\varphi\right)}+1} .
$$


Eq. (2.5) provides a method of determining the mean-field potential, $U$, when using an EoS that does not provide this quantity, e.g., the EoS of ref. [27]. The difference between proton and neutron chemical potentials is directly related to the nuclear symmetry energy, $S(\rho)[30]$ :

$$
\mu_{n}-\mu_{p}=4\left(1-2 Y_{p}\right) S(\rho)=m_{n}-m_{p}+\varphi_{n}-\varphi_{p}+U_{n}-U_{p},
$$

with $Y_{p} \ll 1$ the proton fraction. One can observe that the value of $\Delta U=U_{n}-U_{p}$, and consequently the energy difference between electron antineutrinos and neutrinos is larger for equations of state with a larger value of the symmetry energy.
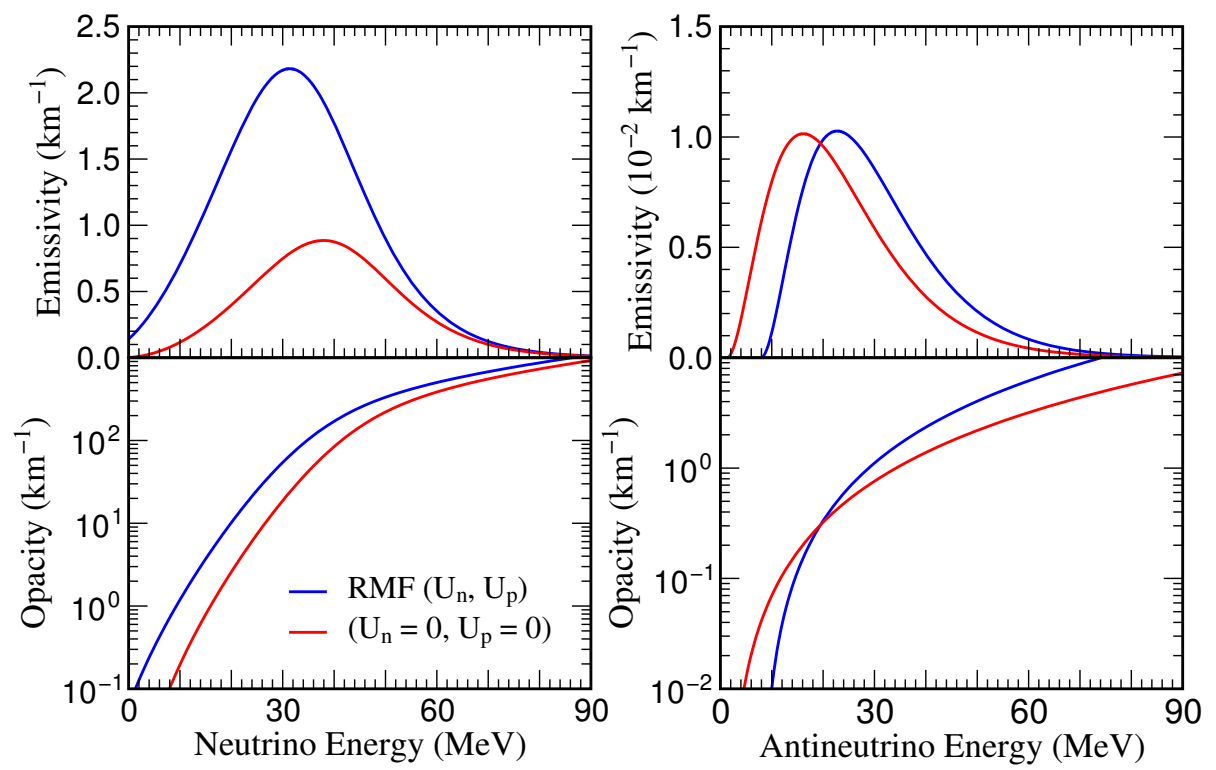

Figure 1: Opacity and emissivity for neutrino (left panels) and antineutrino (right panels), evaluated at conditions $\rho=2.1 \times 10^{13} \mathrm{~g} \mathrm{~cm}^{-3}, T=7.4 \mathrm{MeV}$ and $Y_{e}=0.035$.

Figure 1 shows neutrino and antineutrino opacities and emissivities evaluated at conditions found at the antineutrinosphere for the $18 \mathrm{M}_{\odot}$ model of ref. [15] at $1 \mathrm{~s}$ after bounce. The curves labeled RMF $\left(U_{n}, U_{p}\right)$ include the contribution of the mean-field potentials $U_{n}=-7.6 \mathrm{MeV}$ and $U_{p}=-14.2 \mathrm{MeV}$ [27], while the curves labeled $\left(U_{n}=0, U_{p}=0\right)$ assume a non-interacting gas of nucleons, i.e. neglect the contribution of the potentials but still use chemical potentials as given by the EoS. Due to the presence of the mean-field potentials the effective $Q$-value for electron capture increases with respect to the free case producing neutrinos with substantially lower energy. For the inverse process, neutrino absorption, the opacity is enhanced due to the fact that the produced electron gains an energy $U_{n}-U_{p}$ reducing the final-state Pauli blocking of the electron. The situation is completely analogous to (anti)neutrino emission and absorption on heavy neutron-rich nuclei [31]. Using Eq. (2.4), it can be shown that the opacity for the non-interacting gas, $\chi_{\mathrm{ni}}$, is related to the mean-field opacity, $\chi_{\mathrm{mf}}$ by $\chi_{\mathrm{mf}}(E)=\chi_{\mathrm{ni}}\left(E+U_{n}-U_{p}\right)$. This relationship produces a large enhancement of the neutrino opacity at high densities, $\rho \approx 10^{14}$, where $U_{n}-U_{p} \approx 50 \mathrm{MeV}$ when compared with the non-interacting approximation used in ref. [15]. For antineutrino absorption, positrons are non-degenerate and their spectrum approaches the Boltzmann distribution. Hence, the non-interacting emissivity and mean-field emissivities are related by $j_{\mathrm{mf}}(E)=j_{\mathrm{ni}}\left(E-U_{n}+U_{p}\right)$. 
The mean-field antineutrino opacity is larger at high energies as final-state Pauli blocking of the neutrons becomes less efficient.

\section{Impact in supernova simulations and nucleosynthesis}

To explore the impact that the changes in opacities have on the spectra and luminosities of the emitted neutrinos, we have performed core-collapse supernova simulations based on spherically symmetric radiation hydrodynamics with three-flavor Boltzmann neutrino transport for a $15 \mathrm{M}_{\odot}$ progenitor (see refs $[15,32]$ for additional details). Because spherically symmetric simulations do not result in explosions for such a massive iron-core progenitor, we enhance the neutrino heating rates in the gain region following the scheme of ref. [22]. It results in the onset of explosion at about $350 \mathrm{~ms}$ post bounce. The simulations are evolved from core collapse, through the explosion up to more than 3 seconds after bounce. During core collapse and post-bounce accretion phases, the mean-field potentials, $U_{n}, U_{p}$, are only on the order of several $100 \mathrm{keV}$ in the region of neutrino decoupling, which is located at intermediate densities on the order of $10^{11} \mathrm{~g} \mathrm{~cm}^{-3}$. Hence, their inclusion does not affect the supernova dynamics prior to the explosion.

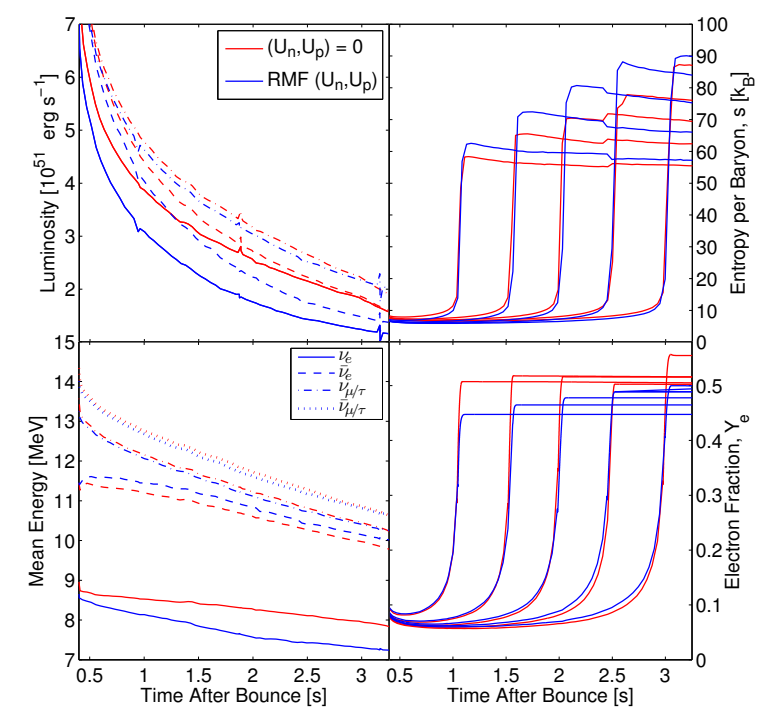

Figure 2: Evolution of the neutrino luminosity (upper left panel) and average energy (lower left panel), proton-to-nucleon ratio, $Y_{e}$, (lower right panel) and entropy per nucleon (upper right panel) for several mass elements ejected from the PNS surface. The curves shown in blue use neutrino opacities computed using the mean-field potentials based on the EoS [27] while they are neglected on the red curves. On the left panels solid lines correspond to electron neutrinos, dashed to electron antineutrinos, dot-dashed to $v_{\mu, \tau}$ and dotted to $\bar{v}_{\mu, \tau}$.

After the onset of the explosion the neutrinospheres move to increasingly higher densities reaching values of the order of $10^{13} \mathrm{~g} \mathrm{~cm}^{-3}$. The left panels of Fig. 2 show the evolution of the luminosity and average neutrino energy for all neutrino flavors. These observables are sampled in a co-moving reference frame at a distance of $1000 \mathrm{~km}$. Using charged-current neutrino opacities that include the mean-field potentials slightly reduces the luminosities for all neutrino flavors. 


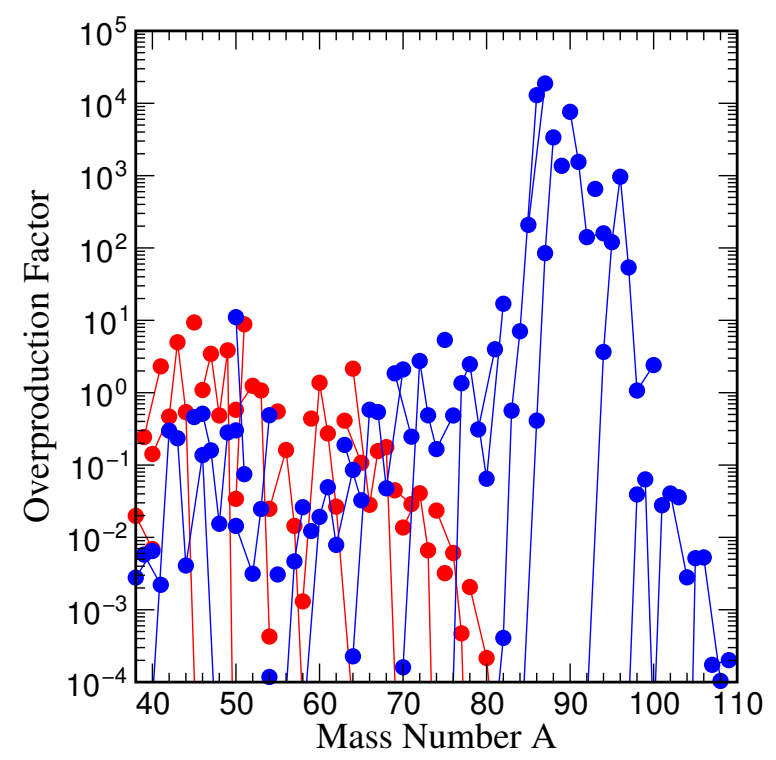

Figure 3: Abundances resulting from nucleosyntheis calculations based on the trajectories shown in figure 2 .

Moreover, as expected from the discussion above, it enhances the differences in luminosities and average energies between electron neutrinos and antineutrinos.

The changes in electron (anti)neutrino spectra and luminosities have important consequences for nucleosynthesis in neutrino-driven winds. The increased difference between average energies of $v_{e}$ and $\bar{v}_{e}$ spectra impacts the $Y_{e}$ of the ejected matter resulting in neutron-rich conditions for the early ejecta and a slight increase in the entropy per nucleon (see fig. 2). In the simulation that neglects the contributions of the mean-field potentials the ejecta are always proton-rich. The decrease in $Y_{e}$ is not large enough to favor an r-process but results in substantial differences in the production of nuclei around $A \sim 90$ (see fig. 3).

We expect that for times larger than those shown in figure 2 the ejecta becomes proton-rich as the average electron neutrino and antineutrino energy approach each other. However, contrary to previous simulations that neglected the contribution of mean-field potentials [21, 22] it will remain a substantial difference between their average energies. This can be related to the relative contribution of charged-current and neutral-current processes to the total neutrino opacity. Fig. 4 shows the position of the neutrinospheres for different neutrino flavors and the opacities as a function of density at $10 \mathrm{~s}$ after bounce based on the data from the $18 \mathrm{M}_{\odot}$ model of ref. [15]. At this time, charged-current processes (red lines) for $\bar{v}_{e}$ have become almost negligible due to the increase in symmetry energy as the neutrinospheres move to high densities. As a consequence the total opacity for $\bar{v}_{e}$ is dominated by neutral current processes. This explains why the spectra of $\bar{v}_{e}$ and $v_{\mu, \tau}$ become very similar at late times as neutral current processes do not differentiate between neutrino flavors. The situation is different for $v_{e}$ neutrinos whose opacity is dominated by charged-current neutrino absorption on neutrons. Notice that this is only the case for opacities that consistently consider the mean-field potential (solid red line). If the mean-field potentials are neglected (dashed red lines) neutrino absorption is largely suppressed. The fact that neutral-current processes dominate for $\bar{v}_{e}$, while charged-current processes dominate for $v_{e}$ is responsible of maintaining a substantial 


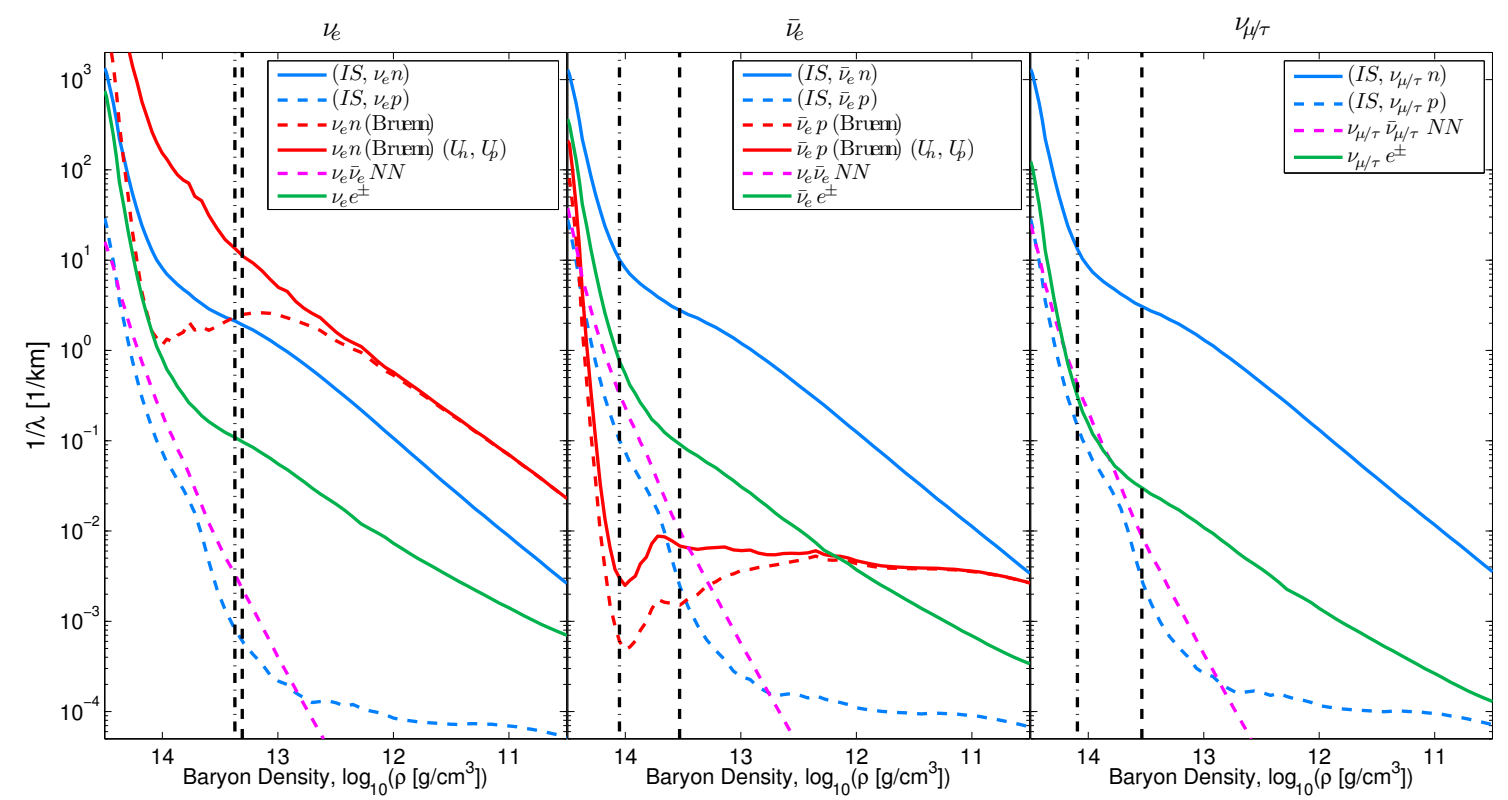

Figure 4: Inverse mean free paths for the individual reactions considered, (IS, $v N)$ : isoenergetic neutrinonucleon scattering, $\left(v_{e} n, \bar{v}_{e} p\right)$ : charged current reactions, $(v \bar{v} N N): N-N-$ Bremsstrahlung), for $v_{e}$ (left panel), $\bar{v}_{e}$ (middle panel) and $v_{\mu / \tau}$ (right panel), based on radial profiles and of a $18.0 \mathrm{M}_{\odot}$ progenitor. The red lines have been computed using equation (2.4) including (solid line) and neglecting (dashed line) mean-field potentials. The vertical black dashed and dash-dotted lines mark the position of the transport and energy spheres. See ref. [15] for additional details.

energy difference between both neutrino species during the whole deleptonization of the protoneutron star.

\section{Conclusions}

We have shown that a treatment of the charged-current (anti)neutrino opacities, that is consistent with the EoS as suggested by [28], has important consequences for the neutrino-spectra evolution during the PNS cooling phase. The most relevant finding is an increased difference between average energies of $v_{e}$ and $\bar{v}_{e}$ that persist during the whole simulation time of 3 seconds after the onset of the explosion. The changes on neutrino spectra are expected to have important consequences for nucleosynthesis, flavor oscillations and neutrino detection on Earth. Our results imply that not only the evolution of the neutrino luminosities [33] but also the spectral differences between $v_{e}$ and $\bar{v}_{e}$ are sensitive to the symmetry energy of nuclear matter. Our simulations are based on neutrino opacities computed using the elastic approximation that neglects momentum exchange between nucleons. They need to be extended to consider the full kinematics [28] of the reaction and many-body correlations $[34,35,36]$ that are expected to become important at later times than those considered in the present study. Furthermore, it is important to explore the sensitivity of the results to different EoS and in particular to EoS that are consistent with recent constrains on the nuclear symmetry energy [37]. 
This work has been partly supported by the Deutsche Forschungsgemeinschaft through contract SFB 634, the Helmholtz International Center for FAIR within the framework of the LOEWE program launched by the state of Hesse, the Helmholtz Association through the Nuclear Astrophysics Virtual Institute (VH-VI-417) and the National Science Foundation under project no. PBBSP2133378 .

\section{References}

[1] H.-T. Janka, K. Langanke, A. Marek, G. Martínez-Pinedo, and B. Müller, Phys. Repts. 442 , 38 (2007).

[2] K. Hirata, T. Kajita, M. Koshiba, M. Nakahata, and Y. Oyama, Phys. Rev. Lett. 58, 1490 (1987).

[3] M. Koshiba, Phys. Repts. 220, 229 (1992).

[4] A. Burrows and J. M. Lattimer, Astrophys. J. 307, 178 (1986).

[5] H. A. Bethe and J. R. Wilson, Astrophys. J. 295, 14 (1985).

[6] B. Müller, H.-T. Janka, and A. Marek, Astrophys. J. 756, 84 (2012).

[7] R. C. Duncan, S. L. Shapiro, and I. Wasserman, Astrophys. J. 309, 141 (1986).

[8] T. A. Thompson, A. Burrows, and B. S. Meyer, Astrophys. J. 562, 887 (2001).

[9] Y.-Z. Qian, Prog. Part. Nucl. Phys. 50, 153 (2003).

[10] H. Duan, G. M. Fuller, and Y. Qian, Ann. Rev. Nucl. Part. Sci. 60, 569 (2010).

[11] S. E. Woosley, D. H. Hartmann, R. D. Hoffman, and W. C. Haxton, Astrophys. J. 356, 272 (1990).

[12] A. Heger, E. Kolbe, W. Haxton, K. Langanke, G. Martínez-Pinedo, and S. E. Woosley, Phys. Lett. B 606, 258 (2005).

[13] P. Banerjee, W. C. Haxton, and Y.-Z. Qian, Phys. Rev. Lett. 106, 201104 (2011).

[14] M. T. Keil, G. G. Raffelt, and H.-T. Janka, Astrophys. J. 590, 971 (2003).

[15] T. Fischer, G. Martínez-Pinedo, M. Hempel, and M. Liebendörfer, Phys. Rev. D 85, 083003 (2012).

[16] S. E. Woosley, J. R. Wilson, G. J. Mathews, R. D. Hoffman, and B. S. Meyer, Astrophys. J. 433, 229 (1994).

[17] K. Takahashi, J. Witti, and H.-T. Janka, Astron. \& Astrophys. 286, 857 (1994).

[18] R. D. Hoffman, S. E. Woosley, and Y.-Z. Qian, Astrophys. J. 482, 951 (1997). 
[19] M. Liebendörfer, A. Mezzacappa, F.-K. Thielemann, O. E. Bronson Messer, W. Raphael Hix, and S. W. Bruenn, Phys. Rev. D 63, 103004 (2001).

[20] R. Buras, M. Rampp, H.-T. Janka, and K. Kifonidis, Astron. \& Astrophys. 447, 1049 (2006).

[21] L. Hüdepohl, B. Müller, H. Janka, A. Marek, and G. G. Raffelt, Phys. Rev. Lett. 104, 251101 (2010).

[22] T. Fischer, S. C. Whitehouse, A. Mezzacappa, F.-K. Thielemann, and M. Liebendörfer, Astron. \& Astrophys. 517, A80 (2010).

[23] C. Fröhlich, G. Martínez-Pinedo, M. Liebendörfer, F.-K. Thielemann, E. Bravo, W. R. Hix, K. Langanke, and N. T. Zinner, Phys. Rev. Lett. 96, 142502 (2006).

[24] J. Pruet, R. D. Hoffman, S. E. Woosley, H.-T. Janka, and R. Buras, Astrophys. J. 644, 1028 (2006).

[25] S. Wanajo, Astrophys. J. 647, 1323 (2006).

[26] J. M. Lattimer and F. D. Swesty, Nucl. Phys. A 535, 331 (1991).

[27] H. Shen, H. Toki, K. Oyamatsu, and K. Sumiyoshi, Nucl. Phys. A 637, 435 (1998).

[28] S. Reddy, M. Prakash, and J. M. Lattimer, Phys. Rev. D 58, 013009 (1998).

[29] S. W. Bruenn, Astrophys. J. Suppl. 58, 771 (1985).

[30] P. Haensel, A. Y. Potekhin, and D. G. Yakovlev, Neutron Stars 1: Equation of State and Structure, vol. 326 of Astrophysics and Space Science Library (Springer, New York, 2007).

[31] K. Langanke and G. Martínez-Pinedo, Rev. Mod. Phys. 75, 819 (2003).

[32] G. Martínez-Pinedo, T. Fischer, A. Lohs, and L. Huther, ArXiv e-prints (2012), 1205.2793, accepted in Phys. Rev. Lett.

[33] L. F. Roberts, G. Shen, V. Cirigliano, J. A. Pons, S. Reddy, and S. E. Woosley, Phys. Rev. Lett. 108, 061103 (2012).

[34] S. Reddy, M. Prakash, J. M. Lattimer, and J. A. Pons, Phys. Rev. C 59, 2888 (1999).

[35] A. Burrows and R. F. Sawyer, Phys. Rev. C 58, 554 (1998).

[36] A. Burrows and R. F. Sawyer, Phys. Rev. C 59, 510 (1999).

[37] J. M. Lattimer and Y. Lim, ArXiv e-prints (2012), 1203.4286. 